\title{
Highly linear and large spring deflection characteristics of a Quasi-Concertina MEMS Device
}

\author{
David Grech $^{\mathrm{a}, \mathrm{b}}$, Kian S. Kiang ${ }^{\mathrm{a}}$, Jurgita Zekonyte ${ }^{\mathrm{b}}$, Martin Stolz ${ }^{\mathrm{b}}$, Robert J.K. Wood ${ }^{\mathrm{b}}$, Harold M. H. Chong \\ ${ }^{a}$ Nano Research Group, Electronics and Computer Science, University of Southampton, Southampton, SO17 1BJ, UK \\ ${ }^{\mathrm{b}}$ National Centre for Advanced Tribology, Faculty of Engineering and the Environment, University of Southampton, Southampton, SO17 \\ 1BJ, UK \\ e-mail: dg5@ecs.soton.ac.uk \\ Telephone and fax numbers: T: +44 (0)23 8059 3127, F: +44 (0)23 80593029
}

\begin{abstract}
In this work a Quasi-Concertina (QC) spring capable of a high linear range, large deflections, high out-ofplane compliance, and low in-plane compliance for MEMS applications is presented. These features are essential for high accuracy out-of-plane measurements such as those required in force-displacement measurements in self-sensing atomic force microscopy (AFM) probes or molecular mass sensors. The spring constant and first mode resonant frequency of the spring was determined analytically and verified numerically. The QC springs were microfabricated using a purposely developed stiction free process. Force-displacement tests on the QC springs have shown them to be in good agreement with the analytical and finite element analysis performed. The measurement results show that the QC springs fabricated have a spring constant of $5.5 \mathrm{~N} / \mathrm{m}$, $0.129 \mathrm{~N} / \mathrm{m}$, and $0.156 \mathrm{~N} / \mathrm{m}$, remain $99 \%$ linear to a deflection of $100 \mu \mathrm{m}, 1080 \mu \mathrm{m}$, and $931 \mu \mathrm{m}$ respectively, and can have a total deflection before fracture of as much as $8000 \mu \mathrm{m}$.
\end{abstract}

Keywords: MEMS, spring, flexure, AFM, force-displacement, Quasi-Concertina

\section{Introduction}

The spring in accelerometers [1], gyroscopes [2], optical mirrors [3], and biosensors [4] is a critical component that affects the overall accuracy, output linearity, and operation range of such micro electromechanical systems (MEMS) devices. The basic spring types used in MEMS are the cantilever, double clamped beam, hammock, crableg, and the folded spring [5]. However, in circumstances where a large linear and travel range is required a serpentine spring [6] is typically used, and a lateral suspension [1] is used when the inplane compliance also needs to be much greater than the out-of-plane compliance. For applications that require the out-of-plane compliance to be much greater than the in-plane compliance, the spring-supported diaphragm [7] would be a suitable choice for small deflections; however, there is still little work done on a flexure type spring that can in addition also give a high linear and large deflection range in the out-of-plane direction.

This paper reports on the design, fabrication, and characterisation of the Quasi-Concertina (QC) spring. The QC spring was developed for a MEMS based static force-displacement sensor for potential applications in self-sensing AFM probes and molecular mass sensors. The QC spring provides a high linear range, large deflection, high out-ofplane compliance and low in-plane compliance.
These features are absent in a single spring type but are essential when high accuracy over large out-ofplane deflection is desirable.

To evaluate the QC spring, the spring constant, first-mode of resonant frequency and the linear range of the device was determined analytically, and numerically. A stiction free release process has been developed for the fabrication of the QC spring. Finally, the characterisation results for the fabricated QC springs are presented and discussed.

\section{Design}

The design of the QC spring, shown in Fig. 1a, is based on using a number of beams in series to increase the linear and deflection range beyond the thickness of beam used [1], and on the mechanical behaviour of beams in which the compliance of a beam in tension or pre-buckling compression is much less than that of the same beam in bending [8]. Using these guidelines, a platform was suspended with beams oriented such that a relatively low spring constant, high linear range, and high deflection range was achieved in the $\mathrm{z}$ out-of-plane direction. The deflection of the platform is restricted in the $\mathrm{x} / \mathrm{y}$ in-plane direction by having interconnected beams on each of the four sides of the platform. In this orientation the beams will go into tension or compression when an inplane load is applied. 


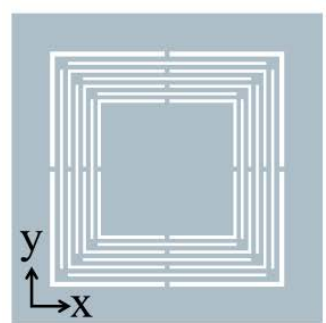

(a)

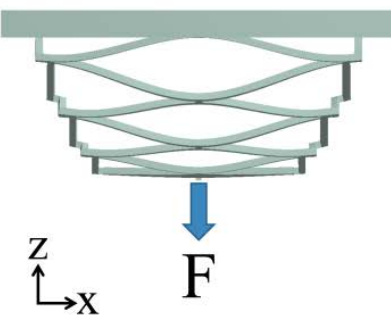

(b)

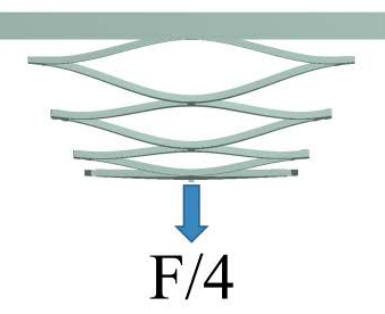

(c)

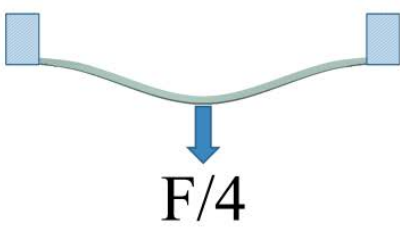

$\mathrm{F} / 4$

Figure 1: (a) The geometry of a QC spring was designed such that multiple beams in series increase the linear and deflection range, increase the z-plane compliance, and oriented to reduce $\mathrm{x} / \mathrm{y}$-plane compliance, (b) FEA simulation of a QC spring deflected

To analytically determine the total spring constant for the device in the $\mathrm{z}$-direction, the platform of the QC spring shown in Fig. 1b is displaced by an applied force, $F$, in the z-direction. The spring is simplified in Fig. 1c by ignoring the beam sets on three of the four sides. Since only one of the four spring sets in parallel now remains, a quarter of the originally applied load is required to achieve the same deflection and therefore the applied load becomes $F / 4$. Further simplification was made by considering each beam in the set equivalent to a double clamped beam (Fig. 1d). Using Bernoulli-Euler beam theory the equation for the spring constant for the beam, $k_{z \text {,beam }}$, was derived as [8]:

$$
k_{z, \text { beam }}=16 E w t^{3} / l^{3} \quad \text { Eq. } 1.1
$$

where $E$ is the Young's modulus (in this work the Si beams were oriented along the $<110>$ direction, $\mathrm{E}=170 \mathrm{GPa}), w$ is the width, $t$ is the thickness, and $l$ is the length of the beam. By calculating the spring constant for each beam in series, the equivalent spring constant for the set was determined using [9]:

$1 / k_{z, \text { series }}=1 / k_{z, \text { beam } 1}+1 / k_{z, \text { beam } 2} \ldots$ Eq. 1.2

and by multiplying by 4 , for the 3 spring sets in parallel ignored earlier, the total spring constant for the flexure is obtained. The values for the platform size, beam thickness, and spring constant for the QC spring samples developed (Table 1) were arbitrarily selected, while the number of beams, beam width, and device size was calculated iteratively using commercial computation software (MATLAB). The results were crosschecked using commercial finite element analysis (FEA) software from ANSYS. FEA was also used to provide the QC spring bending simulations shown in Fig. 1b.

The first-mode resonant frequency for the spring was calculated by considering each beam as a discrete mass connected to a massless spring. Using Newton's second law the equations of motion for the spring was derived, solved numerically using commercial computation software (MATLAB), and verified using FEA.
Table 1

Design parameters of the QC springs fabricated

\begin{tabular}{llll} 
& QC-1 & QC-2 & QC-3 \\
\hline Size & $1.3 \times 1.3 \mathrm{~mm}$ & $2.0 \times 2.0 \mathrm{~mm}$ & $1.8 \times 1.8 \mathrm{~mm}$ \\
Platform size & $0.7 \times 0.7 \mathrm{~mm}$ & $1.1 \times 1.1 \mathrm{~mm}$ & $0.7 \times 0.7 \mathrm{~mm}$ \\
Beams in series & 6 & 17 & 27 \\
Beam width & $24 \mu \mathrm{m}$ & $10 \mu \mathrm{m}$ & $10 \mu \mathrm{m}$ \\
Beam thickness & $4.5 \mu \mathrm{m}$ & $4.5 \mu \mathrm{m}$ & $4.5 \mu \mathrm{m}$ \\
Spring constant & $6 \mathrm{~N} / \mathrm{m}$ & $0.13 \mathrm{~N} / \mathrm{m}$ & $0.16 \mathrm{~N} / \mathrm{m}$
\end{tabular}

\section{Fabrication}

Three QC spring samples with the parameters shown in Table 1 were fabricated using a combined wet and dry release process to realise the samples (Fig. 2). (a)

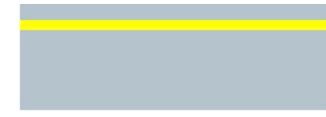

(b)

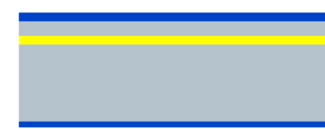

(c)

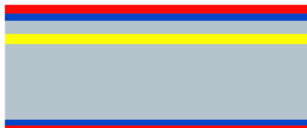

(d)

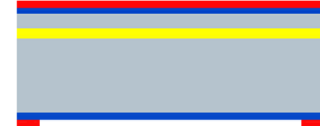

(e)

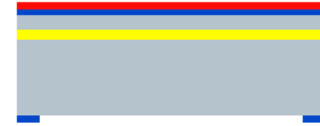

(f)

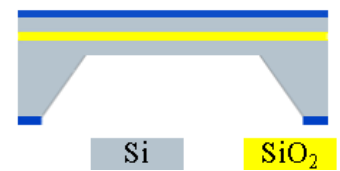

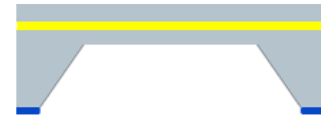

(h)
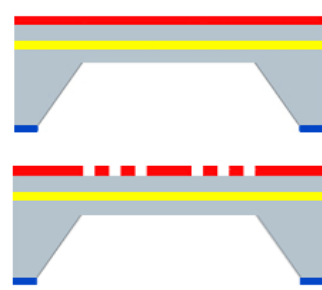

(j)

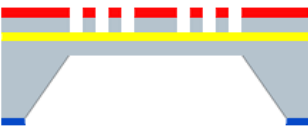

(k)

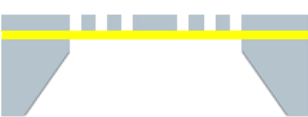

(1)

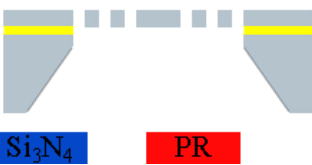

Figure 2: Process flow for the combined wet and dry release process developed for the stiction free fabrication of the QC spring (a) SOI wafer, (b) LPCVD Si3N4, (c) Spin Photoresist (PR), (d) Lithography No. 1, (e) RIE Si3N4 Bottom, (f) KOH etch, (g) RIE Si3N4 - Top, (h) Spin PR, (i) Lithography No. 2, (j) ICP Si - Top, (k) ICP Si - Bottom, (l) RIE SiO2

The fabrication process was performed on silicon on insulator (SOI) wafers with a $4.5 \mu \mathrm{m}$ thick device layer, $2 \mu \mathrm{m}$ BOX layer, and a $575 \mu \mathrm{m}$ handle (Fig. 2a). A low pressure chemical vapour 
deposition (LPCVD) silicon nitride $\left(\mathrm{Si}_{3} \mathrm{~N}_{4}\right)$ mask was deposited on the wafer (Fig. 2b) followed by a standard lithography pattern transfer on to the backside (Fig. 2c-d), and a $\mathrm{Si}_{3} \mathrm{~N}_{4}$ reactive ion etching (RIE) etch (Fig. 2e). A preliminary potassium hydroxide (KOH) wet etch was performed on the handle to semi release the device (Fig. 2f). The $\mathrm{KOH}$ etch was stopped roughly 100 $\mu \mathrm{m}$ short of the buried-oxide (BOX) layer such that the frontside would not buckle which would otherwise make the frontside lithography inaccurate and the wafer too fragile to handle. This was followed by a frontside $\mathrm{Si}_{3} \mathrm{~N}_{4}$ RIE etch, pattern lithography (Fig. 2h-i), and a silicon (Si) inductively coupled plasma (ICP) etch (Fig. 2j). The wafer was diced and the remaining $\mathrm{Si}$ etched from the backside using the ICP (Fig. 2k). Finally the suspended structure was released with a silicon oxide $\left(\mathrm{SiO}_{2}\right)$ RIE etch (Fig. 2l). The main advantage of this process is that stiction is completely avoided. Fig. 3 shows a SEM image of a fabricated QC-1 spring.

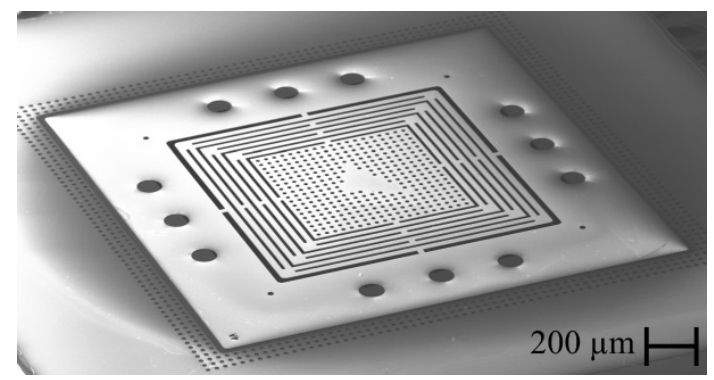

Figure 3: SEM image of a QC-1 microfabricated spring using the combined wet and dry release process

\section{Experiment, and results}

The spring constant was determined by atomic force microscopy (AFM) employing the cantileveron-cantilever method [10]. The setup was modified such that a commercial cantilever was used as the optical lever. The spring constant of the commercial cantilever, $\boldsymbol{k}_{\boldsymbol{c}}$, was determined using the thermal noise method [10, 11], and the cantilever was used to deflect the QC spring to obtain the commercial cantilever deflection versus the total deflection curve. The gradient of the curve, $\boldsymbol{S}_{\boldsymbol{c}}$, was used to calculate the spring constant for the QC spring using:

$$
k=k_{c} /\left(1 / S_{c}-1\right) \cos ^{2} \theta
$$

where $\theta$ is the angle to the horizontal of the commercial cantilever. For accuracy, $k_{c}$ was elected to be similar to that of the QC spring measured [10] and the deflections made were less than the thickness of the commercial cantilever to ensure that the spring constant remained linear.

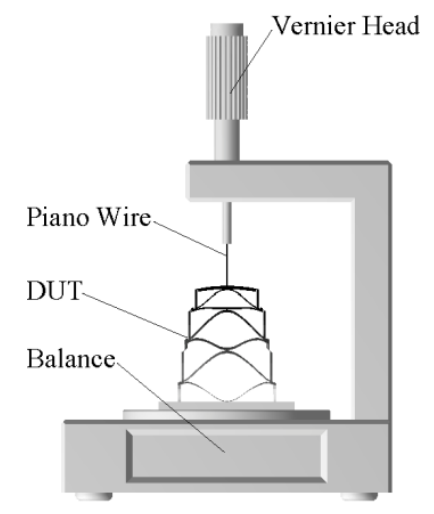

Figure 4: Schematic of the F-D jig used for the mechanical out-of-plane characterisation of the QC-spring. The device under test (DUT) is mounted on a precision balance directly beneath a Vernier micrometre head using a cyanoacrylate adhesive. The suspended structure of the DUT (shown deflected and not to scale) is connected to the Vernier micrometer head with flexible piano wire fixed at both ends using a cyanoacrylate adhesive

For deflections greater than the thickness of the cantilever beam a force-displacement (F-D) jig was used (Fig. 4). The F-D jig was assembled by mounting the QC spring onto a precision balance using a cyanoacrylate adhesive. The balance was positioned directly beneath a linear stage complete with vernier micrometer head. The linear stage was connected to the QC spring with flexible piano wire fixed at both ends using a cyanoacrylate adhesive. Using the vernier micrometer head, the QC spring was pulled away from the balance and the force for each incremental change in displacement was recorded. Initial measurements were conducted to $<50 \%$ of the theoretical maximum deflection and repeated three times following which the test was continued until the QC spring fractured. The data obtained from the AFM cantilever-on-cantilever method, F-D jig, analytical calculations, and FEA analysis was then plotted for each sample (Fig. 5). The first-mode resonant frequencies of the QC springs were determined using a laser Doppler vibrometer. The gradient for the data shown on the forcedisplacement graphs in Fig. 5 represents the spring constant of the QC springs being evaluated. Using the linear range of the F-D jig measurements, the spring constants for the fabricated springs QC-1,2, and 3 was determined to be $5.5 \mathrm{~N} / \mathrm{m}, 0.129 \mathrm{~N} / \mathrm{m}$, and $0.156 \mathrm{~N} / \mathrm{m}$, and remain $99 \%$ linear up to a deflection of $100 \mu \mathrm{m}, 1080 \mu \mathrm{m}$, and $931 \mu \mathrm{m}$ respectively. 


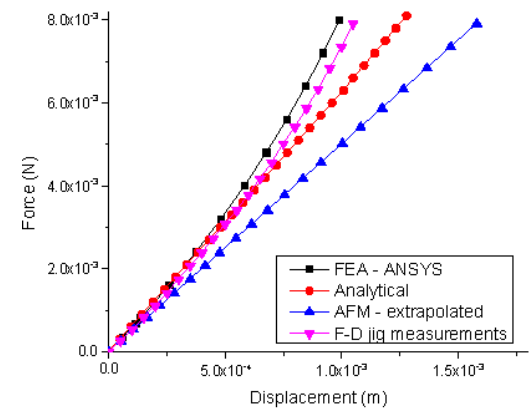

(a)

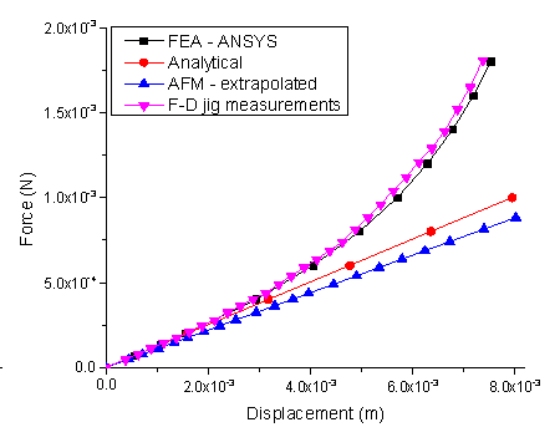

(b)

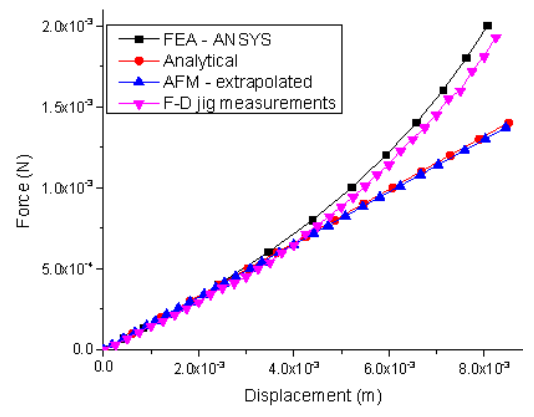

(c)

Figure 5: Force-displacement graphs for (a) QC-1, (b) QC-2, and (c) QC-3 based on analytical, FEA, extrapolated AFM measurement, and F-D jig measurement results

The results also show that a total deflection before fracture of as much as $1000 \mu \mathrm{m}, 7250 \mu \mathrm{m}$, and $8000 \mu \mathrm{m}$ is possible for QC-1,2, and 3 respectively. The complete results are summarised in Table 2

Table 2

Spring constant, linear range, total deflection at fracture, and first-mode resonant frequencies for $\mathrm{QC}-1,2$, and 3 using analytical, FEA, AFM, and F-D jig methods

\begin{tabular}{llll} 
& QC-1 & QC-2 & QC-3 \\
\hline k - Analytical & $6.30 \mathrm{~N} / \mathrm{m}$ & $0.125 \mathrm{~N} / \mathrm{m}$ & $0.165 \mathrm{~N} / \mathrm{m}$ \\
k - FEA & $5.66 \mathrm{~N} / \mathrm{m}$ & $0.166 \mathrm{~N} / \mathrm{m}$ & $0.186 \mathrm{~N} / \mathrm{m}$ \\
k - Measured (AFM) & $4.69 \mathrm{~N} / \mathrm{m}$ & $0.105 \mathrm{~N} / \mathrm{m}$ & $0.162 \mathrm{~N} / \mathrm{m}$ \\
k - Measured (F-D & $5.50 \mathrm{~N} / \mathrm{m}$ & $0.129 \mathrm{~N} / \mathrm{m}$ & $0.156 \mathrm{~N} / \mathrm{m}$ \\
jig) & $100 \mu \mathrm{m}$ & $1080 \mu \mathrm{m}$ & $931 \mu \mathrm{m}$ \\
$99 \%$ Linearity & $350 \mu \mathrm{m}$ & $3125 \mu \mathrm{m}$ & $3558 \mu \mathrm{m}$ \\
$90 \%$ Linearity & $1000 \mu \mathrm{m}$ & $7250 \mu \mathrm{m}$ & $8000 \mu \mathrm{m}$ \\
Total Deflection & $4840 \mathrm{~Hz}$ & $475 \mathrm{~Hz}$ & $654 \mathrm{~Hz}$ \\
$f_{0}$ - Analytical & $4889 \mathrm{~Hz}$ & $535 \mathrm{~Hz}$ & $656 \mathrm{~Hz}$ \\
$f_{0}$ - FEA & $4548 \mathrm{~Hz}$ & $375 \mathrm{~Hz}$ & $672 \mathrm{~Hz}$ \\
$f_{0}$ - Measured & & &
\end{tabular}

\section{Discussion}

Fig. 6 shows the SEM image of a QC spring deflected by $575 \mu \mathrm{m}$. The shape of the deflected beams is analogous to those seen in the FEA simulations and of the double clamped beams in series used in the analytical model.

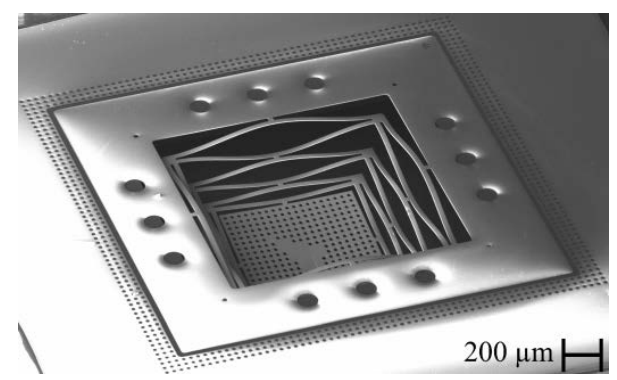

Figure 6: An SEM image of a QC-1 spring deflected by 575 $\mu \mathrm{m}$ to show that the beams bend as shown in the FEA simulations. This also confirms that the beams can be considered to be double-clamped beams in series as was used in the analytical calculations

This verifies the FEA solution and justifies the approach used in the analytical solution. From the graphs in Fig. 5, it is noted that the various methods used to determine the force-displacement characteristics of each spring are in good agreement at low deflections which confirms their validity for determining the behaviour of the springs. This is further reinforced by the agreement of the data obtained for the first-mode of resonance frequency shown in Table 2. However, at increased deflections the analytical data and extrapolated AFM measurements on the force-displacement graphs (Fig. 5) remain linear, while the FEA data and F-D jig measurements show a non-linear increase in the spring constant. This is expected as Bernoulli-Euler elementary beam theory used for the analytical calculations is linear and the AFM force-displacement measurements were extrapolated linearly from the spring constants obtained at low deflections. Unlike the FEA, these methods do not take into account the axial tensions that harden a double clamped beam at higher deflections. This explains why the F-D jig measurements are in better agreement with the FEA data at higher deflections. In Fig. 4a the difference between the gradients of the Analytical and AFM extrapolated data is $26 \%$. This is most likely due to the inherent uncertainty in the cantilever-on-cantilever method used for the AFM measurements which can be as much as $30 \%$ [10]. During the initial force-displacement measurements to $50 \%$ of the theoretical maximum deflection no hysteresis was detected. The out-ofplane results obtained for the QC spring are comparable to what can be achieved using a serpentine spring [6] with the difference that the serpentine spring would lack the low in-plane compliance necessary for high accuracy measurements over large out-of-plane deflections. The QC springs developed in this work were developed for static force-displacement measurements; however, scaling down the size of the device would increase the resonance frequency for improved measuring resolution and scanning rate in dynamic measurement modes.

Future work will include the mechanical characterisation of the in-plane compliance of the QC spring, and the integration of piezoresistors on 
the beams to permit force-displacement selfsensing.

\section{Conclusions}

A novel microfabricated QC spring has been developed for a MEMS based force-displacement sensor suitable for applications such as self-sensing AFM probes and molecular mass sensors. The QC spring provides a high linear range, large deflection, high out-of-plane compliance and low in-plane compliance, which are essential when high accuracy over large deflections is desirable. A combined wet and dry release process which completely avoids stiction has been specifically developed for the fabrication of the springs. The analytical calculations presented in this work are in good agreement with FEA analysis and experimental measurements performed on microfabricated QC springs. The spring constants for the fabricated springs are $5.5 \mathrm{~N} / \mathrm{m}, 0.129 \mathrm{~N} / \mathrm{m}$, and $0.156 \mathrm{~N} / \mathrm{m}$, and remain $99 \%$ linear to a deflection of $100 \mu \mathrm{m}, 1080 \mu \mathrm{m}$, and $931 \mu \mathrm{m}$ respectively. The results also show that a total deflection before fracture of as much as $8000 \mu \mathrm{m}$ is possible.

\section{Acknowledgements}

This project is being funded by the Faculty of Engineering and the Environment (FEE) of the University of Southampton, the European Social Fund (ESF) and the Government of Malta. The author would like to acknowledge the Southampton Nanofabrication Centre at the University of Southampton.

\section{Conflicts of interests}

No conflict of interest is known to exist in this work

\section{References}

[1] W.T. Pike, S. Kumar, J Micromech Microeng, 17 (2007) 1680-1694.

[2] M. Kraft, Meas Control-Uk, 33 (2000) 164-168. [3] Y.H. Bai, J.T.W. Yeow, P. Constantinou, S. Damaskinos, B.C. Wilson, Ieee-Asme T Mech, 15 (2010) 501-510.

[4] J.L. Arlett, E.B. Myers, M.L. Roukes, Nat Nanotechnol, 6 (2011) 203-215.

[5] J.W. Gardner, V.K. Varadan, O.O. Awadelkarim, Microsensors, MEMS, and smart devices, J. Wiley, Chichester ; New York, 2001.

[6] G. Barillaro, A. Molfese, A. Nannini, F. Pieri, J Micromech Microeng, 15 (2005) 736-746.

[7] P.I. Norizan Mohamad, Thurai Vinay, Scientific Research Publishing, 2 (2010) 762-770.

[8] A. Bedford, W.L. Fowler, K. Iynkaran, Engineering mechanics. Statics, SI ed., Pearson/Prentice Hall, Singapore ; London, 2005.

[9] J.J. Allen, Micro electro mechanical system design, Taylor \& Francis/CRC Press, Boca Raton, 2005.

[10] M.-S. Kim, J.-H. Choi, J.-H. Kim, Y.-K. Park, Measurement, 43 (2010) 520-526.

[11] J.L. Hutter, J. Bechhoefer, Rev Sci Instrum, 64 (1993) 3342-3342. 